Homology, Homotopy and Applications, vol.7(1), 2005, pp.39-49

\title{
STRICT MODULES AND HOMOTOPY MODULES IN STABLE HOMOTOPY
}

\author{
JAVIER J. GUTIÉRREZ \\ (communicated by Johannes Huebschmann)
}

\begin{abstract}
Let $R$ be any associative ring with unit and let $H R$ denote the corresponding Eilenberg-Mac Lane spectrum. We show that the category of algebras over the monad $X \mapsto H R \wedge X$ on the homotopy category of spectra is equivalent to the homotopy category associated to a model category of $H R$-module spectra, if the ring $R$ is a field or a subring of the rationals, but not for all rings.
\end{abstract}

\section{Introduction}

Classically, ring spectra and module spectra were defined as objects of the stable homotopy category equipped with suitable structure maps. The stable homotopy category, as described by Adams in [1] , has a smash product which is associative and commutative up to homotopy. The structure maps that define ring spectra and module spectra give rise to diagrams that commute up to homotopy. For a given ring spectrum $E$, the $E$-modules in this sense, together with the $E$-module maps, form a subcategory which can be seen as the Eilenberg-Moore category associated with the monad defined by $X \mapsto E \wedge X$, i.e., the category of algebras over this monad. We call this category the category of homotopy E-modules.

The recent discovery of new structured model categories for stable homotopy, such as the categories of $S$-modules [7] or symmetric spectra [9], equipped with a strictly associative and commutative smash product, allows one to define strict ring spectra (the monoids in the category) and strict module spectra (modules over monoids). The structure maps for these objects give rise to diagrams that truly commute in the model category. Thus, for a strict ring spectrum $E$, we can as well consider the homotopy category of strict $E$-modules, by endowing the category of strict $E$-modules with a model structure as in $[\mathbf{7}]$ or in $[\mathbf{9}]$.

The categories of strict modules have better properties than the categories of homotopy modules. The fibre of an $E$-module map of strict $E$-modules is a strict $E$-module, yet this need not be true for homotopy $E$-modules. The model category of strict $H R$-modules is Quillen equivalent to the category $\mathrm{Ch}(R)$ of unbounded chain complexes of $R$-modules; see [7], [12], [13].

The author was supported by MCyT grants BFM2001-2031 and FP98 16587447.

Received January 12, 2005; published on May 16, 2005.

2000 Mathematics Subject Classification: 55P43, 18E30, 55P42.

Key words and phrases: Ring spectrum, module spectrum, derived category.

(C) 2005, Javier J. Gutiérrez. Permission to copy for private use granted. 
The homotopy category of strict $H R$-modules is not equivalent to the category of homotopy $H R$-modules in general. However, they are equivalent in some special cases, for example when $R=\mathbb{Z}$ (the homotopy $H \mathbb{Z}$-modules are also called stable GEMs). We give a sufficient condition for a ring $R$ in order that there is an equivalence between the homotopy category of strict $H R$-modules and the category of homotopy $H R$-modules. This condition is fulfilled by fields and by subrings of $\mathbb{Q}$.

Acknowledgements. I would like to thank my advisor Carles Casacuberta for his support and encouragement in writing this paper, and also to John Greenlees and Stephan Schwede for many helpful discussions and suggestions during my stay at the Isaac Newton Institute in Cambridge in 2002.

\section{Monads and Eilenberg-Moore categories}

In this section we recall the definition of a monad on a category, and collect some basic results about Eilenberg-Moore categories. These and other facts about monads can be found in $[\mathbf{3}$, Ch. 3], [4, Ch. 4] or [11, Ch. VI].

A monad on a category $\mathcal{C}$ is a triple $\mathbf{T}=(T, \eta, \mu)$ where $T: \mathcal{C} \longrightarrow \mathcal{C}$ is a functor and $\eta: I d_{\mathcal{C}} \longrightarrow T$ and $\mu: T T \longrightarrow T$ are natural transformations such that the following diagrams commute:
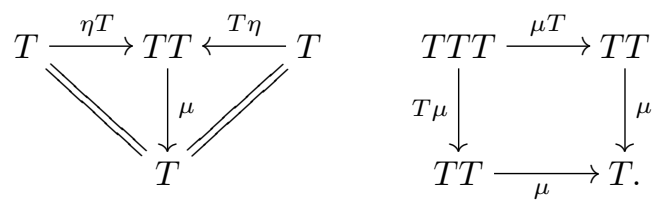

Let $\mathbf{T}=(T, \eta, \mu)$ be a monad on $\mathcal{C}$. An algebra over $\mathbf{T}$ or a $\mathbf{T}$-algebra is a pair $(M, m)$ where $M$ is an object of $\mathcal{C}$ and $m: T M \longrightarrow M$ is a morphism such that the following diagrams commute:
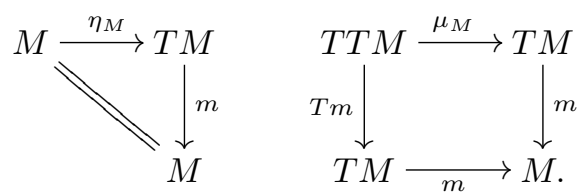

If $(N, n)$ is another $\mathbf{T}$-algebra, then a morphism of $\mathbf{T}$-algebras or a $\mathbf{T}$-morphism $f:(M, m) \longrightarrow(N, n)$ is a morphism $f: M \longrightarrow N$ in $\mathcal{C}$ such that the following diagram commutes:

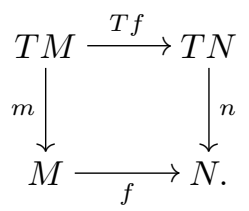

Given a monad $\mathbf{T}=(T, \eta, \mu)$ on a category $\mathcal{C}$, we denote by $\mathcal{C}^{\mathbf{T}}$ the category whose objects are the $\mathbf{T}$-algebras and whose morphisms are the $\mathbf{T}$-morphisms. The category $\mathcal{C}^{\mathbf{T}}$ is called the Eilenberg-Moore category associated with $\mathbf{T}$. 
There is a forgetful functor $U: \mathcal{C}^{\mathbf{T}} \longrightarrow \mathcal{C}$ defined by $U(M, m)=M$ and $U(f)=f$. This functor is faithful and has a left adjoint $F: \mathcal{C} \longrightarrow \mathcal{C}^{\mathbf{T}}$ defined by $F(M)=$ $\left(T M, \mu_{M}\right)$ and $F(f)=T(f)$. This adjunction yields a bijection

$$
\mathcal{C}^{\mathbf{T}}\left(\left(T X, \mu_{X}\right),(Y, m)\right) \cong \mathcal{C}(X, Y)
$$

for any $X \in \mathcal{C}$ and any $\mathbf{T}$-algebra $(Y, m)$.

Given two monads $\mathbf{T}=(T, \eta, \mu)$ and $\mathbf{S}=\left(S, \eta^{\prime}, \mu^{\prime}\right)$ on a category $\mathcal{C}$, a morphism of monads $\mathbf{S} \longrightarrow \mathbf{T}$ is a natural transformation $\lambda: S \longrightarrow T$ such that the following diagrams commute:
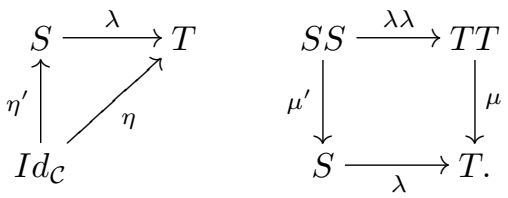

Remark 2.1. Any morphism of monads $\lambda: S \longrightarrow T$ yields a faithful functor between the categories of algebras $Q: \mathcal{C}^{\mathbf{T}} \longrightarrow \mathcal{C}^{\mathbf{S}}$, since any $\mathbf{T}$-algebra has an $\mathbf{S}$-algebra structure via the morphism $\lambda$. Thus, $Q$ is defined as $Q(M, m)=\left(M, m \circ \lambda_{M}\right)$ and $Q(f)=f$. There is a commutative diagram

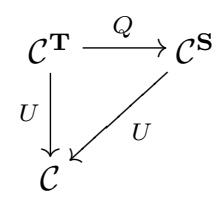

where $U$ is the forgetful functor. This shows that the functor $Q$ is faithful.

Example 2.2. Let $\mathcal{A} b$ be the category of abelian groups and let $R$ be a ring with unit. The functor $R \otimes-: \mathcal{A} b \longrightarrow \mathcal{A} b$ together with the product and the unit of $R$ is a monad on the category of abelian groups. The Eilenberg-Moore category associated with this monad is the category of left $R$-modules.

\section{Stable categories of E-modules}

In this section we describe, for a ring spectrum $E$, the category of strict $E$ modules and the category of homotopy $E$-modules as particular cases of EilenbergMoore categories associated with monads. We will work in the category $S p^{\Sigma}$ of symmetric spectra $[\mathbf{9}]$ as a model category for the stable homotopy category. An object $E$ of $S p^{\Sigma}$ is a ring spectrum if it is equipped with two maps $\mu: E \wedge E \longrightarrow E$ and $\eta: S \longrightarrow E$, where $S$ is the sphere spectrum, such that the following diagrams commute:
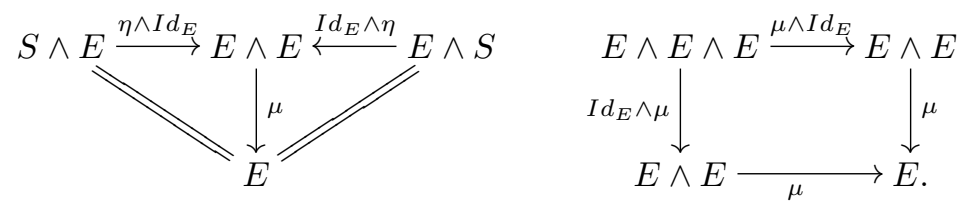
It is said that $E$ is commutative if $\mu \circ \tau=\mu$ where $\tau: E \wedge E \longrightarrow E \wedge E$ is the twist map. Given a ring spectrum $E \in S p^{\Sigma}$, an $E$-module spectrum is a pair $(M, m)$ with $M \in S p^{\Sigma}$ and $m: E \wedge M \longrightarrow M$ such that the following diagrams commute:
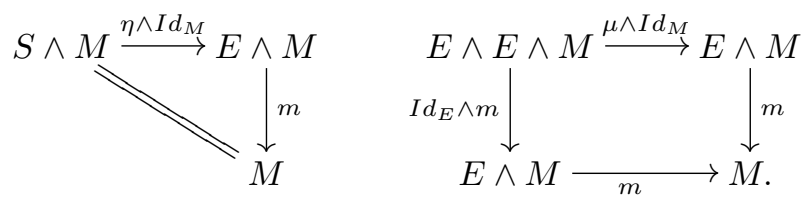

Example 3.1. If $R$ is an associative ring with unit and $M$ is a left $R$-module, then the Eilenberg-Mac Lane spectrum $H R$ is a ring spectrum and the spectrum $H M$ is an $H R$-module spectrum. The structure maps of $H R$ and $H M$ come from the product and the unit of $R$, and from the structure homomorphism of $M$ as an $R$-module.

A map of E-modules or an E-module map $f:(M, m) \longrightarrow(N, n)$ is a map $f: M \longrightarrow N$ such that the following diagram commutes:

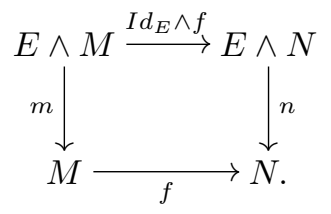

For any ring spectrum $(E, \eta, \mu)$ we can consider the functor $E \wedge-: S p^{\Sigma} \longrightarrow$ $S p^{\Sigma}$. This functor sends any $X$ to an $E$-module spectrum $E \wedge X$. The natural transformations $\eta \wedge I d: I d_{S p^{\Sigma}} \longrightarrow E \wedge-$ and $\mu \wedge I d: E \wedge E \wedge-\longrightarrow E \wedge-$ form a monad on the category of symmetric spectra, by the commutativity of (3.1). The Eilenberg-Moore category associated with the monad $(E \wedge-, \eta \wedge I d, \mu \wedge I d)$ will be denoted by E-mod and called the category of strict E-modules. By [13], this category admits a model category structure. If $H o(E-m o d)$ is the corresponding homotopy category, and $M$ and $N$ are objects in this category, we denote by $[M, N]_{E-\bmod }$ the group of morphisms between $M$ and $N$ in $H o(E-m o d)$. Thus, (1) yields a bijection

$$
E-\bmod (E \wedge M, N) \cong S p^{\Sigma}(M, N)
$$

for any $E$-module spectrum $N$ and any $M$. This bijection does not induce a bijection of homotopy classes of maps in general, as shown in Corollaries 4.5 and 4.6.

Now, for a ring spectrum $E \in S p^{\Sigma}$, consider the monad $(E \wedge-, \eta \wedge I d, \mu \wedge I d)$ on the homotopy category $\mathrm{Ho}\left(S p^{\Sigma}\right)$. The Eilenberg-Moore category associated with this monad will be called the category of homotopy E-modules, and denoted by $E$-hmod. If $M$ and $N$ are objects in $E$-hmod, we denote by $[M, N]_{E-h m o d}$ the group of morphisms between them in the Eilenberg-Moore category. If $N$ is a homotopy $E$-module and $M$ is any spectrum, then the bijection (1) gives an isomorphism

$$
[E \wedge M, N]_{E-h \text { mod }} \cong[M, N] \text {. }
$$

Note that the objects in $E$-hmod are $E$-module spectra in the traditional sense, i.e., endowed with structure maps for which the diagrams (3) and (4) commute up to homotopy. Thus, every strict $E$-module is a homotopy $E$-module. 


\section{Homotopy modules and derived categories}

The categories E-hmod and $H o(E-m o d)$, defined in the previous section, are very different in general. In this section we compare these two categories in the case where $E$ is the ring spectrum $H R$, for some associative ring $R$ with unit. In what follows $R$-modules will be left modules.

The derived category $\mathcal{D}(R)$ of the ring $R$ is defined as the homotopy category of $\mathrm{Ch}(R)$, the model category of unbounded chain complexes of $R$-modules; see [8]. The weak equivalences are the quasi-isomorphisms, i.e., the maps inducing isomorphisms in homology. If $E$ is any $R$-module, we will denote by $E[k]$ the chain complex

$$
\cdots \longrightarrow 0 \longrightarrow 0 \longrightarrow E \longrightarrow 0 \longrightarrow 0 \longrightarrow \cdots
$$

where $E$ is located in dimension $k$. If $A$ and $B$ are two $R$-modules, then the following holds:

$$
\mathcal{D}(R)(A[0], B[k])=\operatorname{Ext}_{R}^{k}(A, B) ;
$$

see [14, Ch. 10] for a useful description of the derived category.

The projective dimension $\operatorname{pd}(A)$ of an $R$-module $A$ is the minimum integer $n$ (if it exists) such that there is a projective resolution of $A$ of length $n$,

$$
0 \longrightarrow P_{n} \longrightarrow P_{n-1} \longrightarrow \cdots \longrightarrow P_{0} \longrightarrow A \longrightarrow 0 .
$$

If no such integer exists, we say that $\operatorname{pd}(A)=\infty$. The global dimension of a ring $R$ is defined as $\operatorname{gd}(R)=\sup _{A \in R \text {-mod }}\{\operatorname{pd}(A)\}$. For example, $\operatorname{gd}(\mathbb{Z})=1, \operatorname{gd}\left(\mathbb{Z} / p^{2}\right)=\infty$ if $p$ is a prime, $\operatorname{gd}\left(R\left[x_{1}, \ldots, x_{n}\right]\right)=\operatorname{gd}(R)+n$.

The rings $R$ with $\operatorname{gd}(R)=0$ are called semisimple. All fields and finite direct products of fields are semisimple rings. In general, $\operatorname{gd}(R)=0$ if and only if $R$ is a finite direct product of matrix rings over division rings, by the Wedderburn-Artin Theorem; see $[\mathbf{2}, \S 13]$, for example. Thus, if $R$ is commutative, then $\operatorname{gd}(R)=0$ if and only if $R$ is a finite direct product of fields.

The groups $\mathrm{Ext}_{R}^{k}$ are closely related with the global dimension of the ring $R$. A classical theorem in homological algebra states that $\operatorname{gd}(R)=k$ if and only if $\operatorname{Ext}_{R}^{i}(A, B)=0$ for $i>k$ and all $R$-modules $A$ and $B$; see [14].

Proposition 4.1. If $\operatorname{gd}(R) \leqslant 1$, then any chain complex of $R$-modules

$$
\mathbf{C}: \cdots \longrightarrow C_{n} \stackrel{d_{n}}{\longrightarrow} C_{n-1} \stackrel{d_{n-1}}{\longrightarrow} \cdots \longrightarrow C_{0} \stackrel{d_{0}}{\longrightarrow} C_{-1} \longrightarrow \cdots
$$

is weakly equivalent, and hence isomorphic in $\mathcal{D}(R)$, to $\oplus_{k \in \mathbb{Z}} H_{k}(\mathbf{C})[k]$.

Proof. If $\operatorname{gd}(R)=0$, then there exists a map $\widetilde{p}_{k}: H_{k}(\mathbf{C}) \longrightarrow \operatorname{ker} d_{k} \subset C_{k}$ such that $\pi \circ \widetilde{p}_{k}=i d$, where $\pi$ denotes the projection ker $d_{k} \longrightarrow H_{k}(\mathbf{C})$, since every $R$-module is projective. Hence, for each $k \in \mathbb{Z}$ we have a map

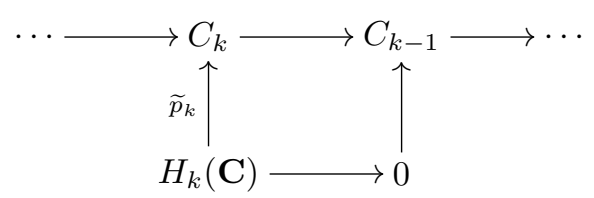


and this yields a map of chain complexes $\phi: \oplus_{k \in \mathbb{Z}} H_{k}(\mathbf{C})[k] \longrightarrow \mathbf{C}$ inducing an isomorphism in homology. For the case $\operatorname{gd}(R)=1$, let $R_{k} \stackrel{i_{k}}{\longrightarrow} F_{k} \stackrel{p_{k}}{\longrightarrow} H_{k}(\mathbf{C})$ be a projective resolution of the $k$-th homology group $H_{k}(\mathbf{C})$. Take $\mathbf{A}_{k}$ to be the complex $\cdots \rightarrow 0 \rightarrow R_{k} \rightarrow F_{k} \rightarrow 0 \rightarrow \cdots$ with $F_{k}$ in dimension $k$ and $R_{k}$ in dimension $k+1$. Now we construct a map from $\oplus_{k \in \mathbb{Z}} \mathbf{A}_{k}$ to $\mathbf{C}$ inducing an isomorphism in homology. For each $k \in \mathbb{Z}$, we have the following diagram:

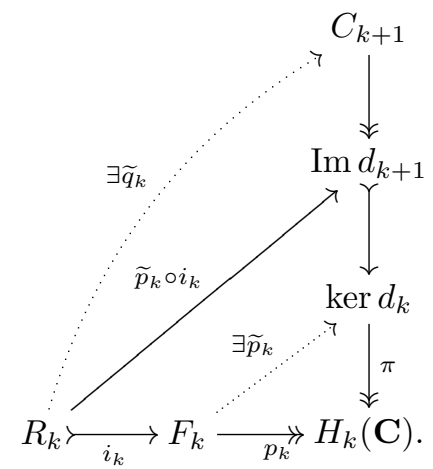

Since $F_{k}$ is projective and $\pi$ is surjective, there exists a map $\widetilde{p}_{k}: F_{k} \longrightarrow \operatorname{ker} d_{k} \subset C_{k}$ closing the diagram. The map $\widetilde{p}_{k} \circ i_{k}$ lifts to $\operatorname{Im} d_{k+1}$ because $\widetilde{p}_{k} \circ i_{k} \subset \operatorname{ker} \pi=$ $\operatorname{Im} d_{k+1}$. Again, $R_{k}$ is projective and the map $C_{k+1} \rightarrow \operatorname{Im} d_{k+1}$ is surjective, hence there exists a map $\widetilde{q}_{k}: R_{k} \longrightarrow C_{k+1}$ closing the diagram. For each $k \in \mathbb{Z}$, we have defined maps $\widetilde{p}_{k}$ and $\widetilde{q}_{k}$

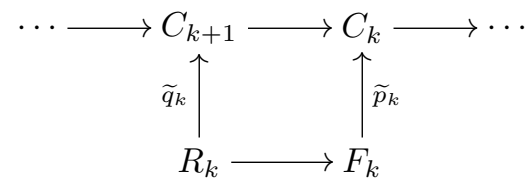

and this yields a map $\phi: \oplus_{k \in \mathbb{Z}} \mathbf{A}_{k} \longrightarrow \mathbf{C}$ that is a quasi-isomorphism. Since the complex $\mathbf{A}_{k}$ is quasi-isomorphic to $H_{k}(\mathbf{C})[k]$, we have that $\oplus_{k \in \mathbb{Z}} H_{k}(\mathbf{C})[k]$ and $\mathbf{C}$ are quasi-isomorphic.

Remark 4.2. Note that Proposition 4.1 does not hold if $\operatorname{gd}(R)>1$. Suppose that $\operatorname{gd}(R)=k>1$ and consider a nonzero element $\xi$ in $\operatorname{Ext}_{R}^{k}(M, N)$, where $M$ and $N$ are $R$-modules. This element $\xi$ can be represented by an extension of modules

$$
0 \longrightarrow N \longrightarrow E_{k} \longrightarrow \cdots \longrightarrow E_{1} \longrightarrow M \longrightarrow 0,
$$

where $E_{1}, \ldots, E_{k}$ are free [10, Corollary III.6.5]. Take now the chain complex

$$
\mathbf{E}: \cdots \longrightarrow 0 \longrightarrow E_{k} \longrightarrow E_{k-1} \longrightarrow \cdots \longrightarrow E_{1} \longrightarrow 0 \longrightarrow \cdots
$$

where $E_{1}$ is in dimension 0 . This complex has homology only in dimensions 0 and $k-$ 1 , namely $H_{0}(\mathbf{E})=M$ and $H_{k-1}(\mathbf{E})=N$. But if this complex is quasi-isomorphic to $M[0] \oplus N[k-1]$ then $\xi=0$, because $E_{1}, \ldots, E_{k}$ are free and hence there exists a quasi-isomorphism from the first complex to the second one, and therefore a 
commutative diagram

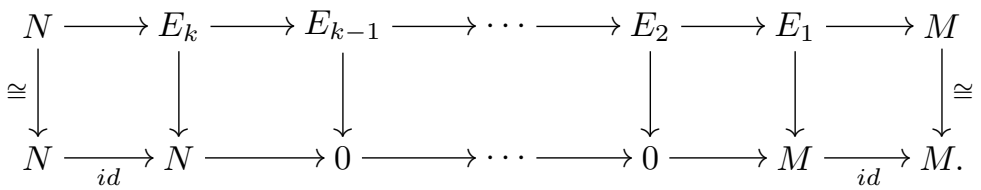

The following result was first proved in $[\mathbf{1 2}]$. A recent generalization can be found in $[\mathbf{1 3}]$.

Theorem 4.3. For any ring $R$ there is a Quillen equivalence between the model category of unbounded chain complexes of $R$-modules and the model category of (strict) $H R$-modules. This equivalence induces an equivalence between the homotopy categories $\mathcal{D}(R)$ and $H o(H R$-mod) that sends each $H R$-module $M$ to a chain complex $\mathbf{C}$ such that $H_{k}(\mathbf{C}) \cong \pi_{k}(M)$ for every $k \in \mathbb{Z}$.

The objects of the category $H R$-hmod are precisely the stable $R$-GEMs and have been studied in [6, Section 5]. Recall that a spectrum $E \in H o\left(S p^{\Sigma}\right)$ is a stable $R$ GEM if $E \simeq \vee_{k \in \mathbb{Z}} \Sigma^{k} H A_{k}$ where each $A_{k}$ is an $R$-module. In the case $R=\mathbb{Z}$, by [6, Proposition 5.3], any $H \mathbb{Z}$-module is isomorphic in $H \mathbb{Z}$-hmod to $\vee_{k \in \mathbb{Z}} \Sigma^{k} H A_{k}$, where $A_{k} \cong \pi_{k}(M)$. Hence, if $M, N \in H \mathbb{Z}$-hmod, and $M \simeq \vee_{k \in \mathbb{Z}} \Sigma^{k} H A_{k}$ and $N \simeq \vee_{j \in \mathbb{Z}} \Sigma^{j} H B_{j}$, then

$$
[M, N]_{H \mathbb{Z}-h \text { mod }}=\prod_{k \in \mathbb{Z}} \prod_{j \in \mathbb{Z}}\left[H A_{k}, \Sigma^{j-k} H B_{j}\right]_{H \mathbb{Z}-h m o d}
$$

since the natural map $\vee_{k \in \mathbb{Z}} \Sigma^{k} H A_{k} \longrightarrow \prod_{k \in \mathbb{Z}} \Sigma^{k} H A_{k}$ is an equivalence in this particular case. Thus, the study of morphisms in $H \mathbb{Z}$-hmod amounts to the study of $\left[H A, \Sigma^{k} H B\right]_{H \mathbb{Z}-h m o d}$. These abelian groups have already been described in $[6$, Section 5], as follows:

Proposition 4.4. For all abelian groups $A$ and $B$, the following holds:

$$
\begin{aligned}
& {[H A, H B]_{H \mathbb{Z}-h \text { mod }} \cong \operatorname{Hom}(A, B),} \\
& {[H A, \Sigma H B]_{H \mathbb{Z}-h \text { mod }} \cong \operatorname{Ext}(A, B), \text { and }} \\
& {\left[H A, \Sigma^{k} H B\right]_{H \mathbb{Z}-h \text { mod }}=0 \text { if } k \neq 0,1 .}
\end{aligned}
$$

Proof. The left adjoint of the monad given by $H \mathbb{Z} \wedge-$ yields an isomorphism $\left[M A, \Sigma^{k} H B\right] \cong\left[H A, \Sigma^{k} H B\right]_{H \mathbb{Z}-h m o d}$ because $H A \simeq H \mathbb{Z} \wedge M A$ where $M A$ denotes a Moore spectrum for the abelian group $A$; see [1]. Now use the exact sequence

$$
0 \rightarrow \operatorname{Ext}\left(A, \pi_{k+1} X\right) \rightarrow\left[\Sigma^{k} M A, X\right] \rightarrow \operatorname{Hom}\left(A, \pi_{k} X\right) \rightarrow 0
$$

in the case $X=H B$.

Corollary 4.5. Given any ring $R$ and $R$-modules $A$ and $B$, if $k \neq 0,1$, then $\left[H A, \Sigma^{k} H B\right]_{H R-h m o d}=0$.

Proof. The inclusion $\mathbb{Z} \hookrightarrow R$ provides a natural transformation $H \mathbb{Z} \wedge-\longrightarrow H R \wedge-$ and a morphism of monads. The result follows from Remark 2.1. 
Corollary 4.6. There is no equivalence of categories between the categories $H o(H R$-mod $)$ and $H R$-hmod if $\operatorname{gd}(R)>1$.

Proof. Suppose that there exists an equivalence between the categories $H R$-hmod and $H o(H R$-mod $)$. Then for any $R$-modules $A, B$ and any $k \in \mathbb{Z}$, we have that

$$
\left[H A, \Sigma^{k} H B\right]_{H R-h \text { mod }} \cong\left[H A, \Sigma^{k} H B\right]_{H R-\bmod }=\operatorname{Ext}_{R}^{k}(A, B)
$$

by Theorem 4.3. But $\left[H A, \Sigma^{k} H B\right]_{H R \text {-hmod }}=0$ for $k \neq 0,1$ by Corollary 4.5 , and this is a contradiction since $\operatorname{gd}(R)>1$.

We will now discuss $\left[H A, \Sigma^{k} H B\right]_{H R-h m o d}$ in the cases $k=0$ and $k=1$, for any $\operatorname{ring} R$. The following proposition generalizes Proposition 4.4 for any ring $R$ in the case $k=0$.

Proposition 4.7. For any ring $R$ and all $R$-modules $A$ and $B$, the correspondence $f \mapsto \pi_{0}(f)$ yields a natural isomorphism

$$
[H A, H B]_{H R-h m o d} \cong \operatorname{Hom}_{R}(A, B) .
$$

Proof. Let $f: H A \longrightarrow H B$ be any map. Recall that $H A$ and $H B$ are $H R$-modules because $A$ and $B$ are $R$-modules. The map $f$ will be a map in $H R$-hmod if the diagram

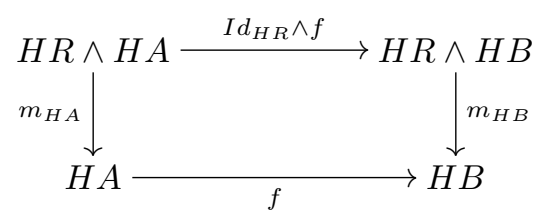

commutes up to homotopy. We can define a map $\Phi:[H A, H B] \longrightarrow[H R \wedge H A, H B]$ by $\Phi(f)=f \circ m_{H A}-m_{H B} \circ\left(I d_{H R} \wedge f\right)$. Then $f$ is a map in $H R$-hmod if and only if $f \in \operatorname{ker} \Phi$. But $[H A, H B] \cong \operatorname{Hom}(A, B)$ and $[H R \wedge H A, H B] \cong \operatorname{Hom}(R \otimes A, B)$. The map $f$ is in $\operatorname{ker} \Phi$ if and only if $f(r a)=r f(a)$ for all $r \in R$ and $a \in A$, and this is the same as stating that $f \in \operatorname{Hom}_{R}(A, B)$.

The case $k=1$ is more involved. Although we can give a description of $[H A, \Sigma H B]_{H R-h \text { mod }}$ as the kernel of a map $\Phi:[H A, \Sigma H B] \longrightarrow[H R \wedge H A, \Sigma H B]$, as in the proof of Proposition 4.7, and

$$
[H A, \Sigma H B] \cong \operatorname{Ext}(A, B) \text { and }[H R \wedge H A, \Sigma H B] \cong \operatorname{Ext}(R \otimes A, B),
$$

it turns out that $[H A, \Sigma H B]_{H R \text {-hmod }} \neq \operatorname{Ext}_{R}(A, B)$ in general. Indeed, suppose that $[H A, \Sigma H B]_{H R \text {-hmod }} \cong \operatorname{Ext}_{R}(A, B)$. Then, by Remark 2.1 and Proposition 4.4, there would be an injective map

$$
\operatorname{Ext}_{R}(A, B) \longleftrightarrow \operatorname{Ext}(A, B)
$$

for any ring $R$ and all $R$-modules $A$ and $B$, and this is not true. The following counterexample for a ring $R$ of global dimension one was pointed out to us by Jérôme Scherer. 
Example 4.8. Let $R=\mathbb{Q}[x], A=\mathbb{Q}[x] /\left(x^{n}\right)$ and $B=\mathbb{Q}$. Then $\operatorname{Ext}_{R}(A, B) \neq 0$ because the exact sequence

$$
\mathbb{Q} \succ \longrightarrow \mathbb{Q}[x] /\left(x^{n+1}\right) \longrightarrow \mathbb{Q}[x] /\left(x^{n}\right)
$$

does not split. If this splitting did exist, then $x^{n}=0$ in $\mathbb{Q}[x] /\left(x^{n+1}\right)$, which is a contradiction. On the other hand, $\operatorname{Ext}(A, B)=0$ as abelian groups because $\mathbb{Q}$ is divisible.

Note that this example shows that, for the ring $R=\mathbb{Q}[x]$, which has global dimension 1 , there is no possible equivalence of categories between $H o(H R-m o d)$ and $H R$-hmod.

\section{An equivalence of categories}

In this section we study for which rings $R$ there is an equivalence of categories between $H o(H R$-mod $)$ and $H R$-hmod. As we have seen, Corollary 4.6 states that there is no possible equivalence if $\operatorname{gd}(R)>1$. But not all rings of global dimension one yield such an equivalence, as illustrated by Example 4.8. However, as we next show, the equivalence holds if the ring $R$ is a field or $R$ is a subring of the rationals.

Proposition 5.3 of [6] can be extended to the case of $H R$-modules when $R$ is a field or $R$ is a torsion free solid ring. For these rings, equality (5) also holds if one replaces $\mathbb{Z}$ by $R$. If $R$ is a field, then every $R$-module splits as a direct sum of copies of $R$, and hence

$$
[H A, \Sigma H B]_{H R-h \bmod } \cong\left[\vee_{i} H R, \Sigma H B\right]_{H R-h \bmod } \cong \prod_{i}[S, \Sigma H B]=0 .
$$

A ring $R$ is solid if the multiplication induces an isomorphism $R \otimes R \cong R$, where the tensor product is over $\mathbb{Z}$. Solid rings were introduced in [5]. If $R$ is solid, then in particular $R \otimes A \cong A$ whenever $A$ is an $R$-module. This implies that, if $R$ is solid, then

$$
\operatorname{Hom}_{R}(A, B) \cong \operatorname{Hom}(A, B) \text { and } \operatorname{Ext}_{R}(A, B) \cong \operatorname{Ext}(A, B) .
$$

If $R$ is torsion-free, then $H R \wedge M A \simeq H(R \otimes A)$ for any $R$-module $A$. If a ring $R$ satisfies these two conditions, then $[H A, \Sigma H B]_{H R-h m o d}=\operatorname{Ext}_{R}(A, B)$.

Lemma 5.1. If $R$ is a torsion-free solid ring of global dimension one, then $R$ is a subring of the rationals.

Proof. This follows from the classification of solid rings (see [5]).

Theorem 5.2. If $R$ is a field or $R$ is a subring of the rationals, then there is an equivalence of categories between $H o(H R$-mod $)$ and $H R$-hmod.

Proof. We construct a functor $\Phi: H R$-hmod $\longrightarrow \mathcal{D}(R)$ that is an equivalence of categories. It will be enough to define the functor on objects of the form $\Sigma^{i} H A$, since any $M \in H R$-hmod is isomorphic to $\vee_{k \in \mathbb{Z}} \Sigma^{k} H A_{k}$ in $H R$-hmod, and on morphisms of the form $f: H A \longrightarrow \Sigma^{k} H B$ in the cases $k=0$ and $k=1$, by equality (5). If 
$\phi$ is an equivalence of categories, $H o(H R$-mod $) \simeq H R$-hmod by Theorem 4.3. We consider separately the case of a field and of a subring of the rationals.

If $R$ is a field, then $\operatorname{gd}(R)=0$ and hence $\operatorname{Ext}_{R}(A, B)=0$. We define $\Phi\left(\Sigma^{k} H A\right)=$ $A[k]$ and, thus, if $M \in H R$-hmod is such that $M \simeq \vee_{k \in \mathbb{Z}} \Sigma^{k} H A_{k}$, then $\Phi(M)=$ $\oplus_{k \in \mathbb{Z}} A_{k}[k]$. Thus, for a map $f: H A \longrightarrow H B$ we define $\Phi(f)=\pi_{0}(f)$, the corresponding map between $A[0]$ and $B[0]$. Now, $\Phi$ is a functor and it is full and faithful. Moreover, every object in $\mathcal{D}(R)$ lies in the image of $\Phi$ up to isomorphism by Proposition 4.1, so it is an equivalence of categories.

If $R$ is a subring of $\mathbb{Q}$, we define $\Phi\left(\Sigma^{k} H A\right)=\mathbf{P}_{k}(A)$ where $\mathbf{P}_{k}(A)$ is the complex

$$
\cdots \longrightarrow 0 \longrightarrow R_{k} \longrightarrow F_{k} \longrightarrow 0 \longrightarrow \cdots
$$

with $F_{k}$ in dimension $k$, and $R_{k} \rightarrow F_{k} \rightarrow A$ is a projective resolution of $A$. If $M \simeq$ $\vee_{k \in \mathbb{Z}} \Sigma^{k} H A_{k}$, then $\Phi(M)=\oplus_{k \in \mathbb{Z}} \mathbf{P}_{k}\left(A_{k}\right)$. A map $f \in[H A, H B]_{H R-h \text { mod }}$ corresponds to a morphism of $R$-modules from $A$ to $B$ and hence lifts to a map $\widetilde{f}$ between the projective resolutions of $A$ and $B$

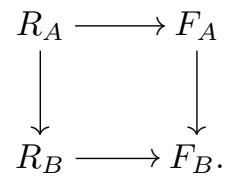

This yields a map from the complex $\mathbf{P}_{0}(A)=\Phi(H A)$ to $\mathbf{P}_{0}(B)=\Phi(H B)$. We define $\Phi(f)=\widetilde{f}$.

Similarly, a map $g \in[H A, \Sigma H B]_{H R \text {-hmod }}$ lifts to a map $\widetilde{g}$ between the complexes $\mathbf{P}_{0}(A)=\Phi(H A)$ and $\mathbf{P}_{1}(B)=\Phi(\Sigma H B)$,

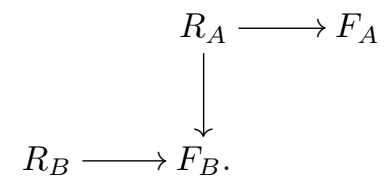

We define $\Phi(g)=\widetilde{g}$. The Yoneda product

$$
\operatorname{Ext}_{R}^{i}(B, C) \otimes \operatorname{Ext}_{R}^{j}(A, B) \stackrel{Y}{\longrightarrow} \operatorname{Ext}_{R}^{i+j}(A, C)
$$

makes $\Phi$ a functor. This functor is full and faithful by Proposition 4.7 and because $[H A, \Sigma H B]_{H R \text {-hmod }}=\operatorname{Ext}_{R}(A, B)$. By Proposition 4.1, every object in $\mathcal{D}(R)$ lies in the image of $\Phi$ up to isomorphism, so it is an equivalence of categories.

\section{References}

[1] J. F. Adams, Stable Homotopy and Generalised Homology. Chicago Lectures in Mathematics. University of Chicago Press, Chicago, London, 1974.

[2] F. W. Anderson and K. R. Fuller, Rings and Categories of Modules. Graduate Texts in Mathematics, vol. 13. Springer-Verlag, New York, 1974.

[3] M. Barr and C. Wells, Toposes, Triples and Theories. Grundlehren der mathematischen Wissenschaften, Band 278. Springer-Verlag, New York, 1985. 
[4] F. Borceux, Handbook of Categorical Algebra. Basic Category Theory. Encyclopedia of Mathematics and its Applications, 50. Cambridge University Press, Cambridge, 1994.

[5] A. K. Bousfield and D. M. Kan, The core of a ring. J. Pure Appl. Algebra 2 (1972), pp. 73-81.

[6] C. Casacuberta J. J. Gutiérrez, Homotopical localizations of module spectra. To appear in Trans. Amer. Math. Soc..

[7] A. D. Elmendorf, I. Kriz, M. A. Mandell J. P. May, Rings, Modules, and Algebras in Stable Homotopy Theory. Mathematical Surveys and Monographs, 47. Amer. Math. Soc., Providence, 1997.

[8] M. Hovey, Model Categories. Mathematical Surveys and Monographs, vol. 63. Amer. Math. Soc., Providence, 1999.

[9] M. Hovey, B. Shipley J. Smith, Symmetric spectra. J. Amer. Math. Soc. 13 (2000), no. 1, pp. 149-208.

[10] S. Mac Lane, Homology. Die Grundlehren der mathematischen Wissenschaften, Band 114. Springer-Verlag, New York, 1971

[11] S. Mac Lane, Categories for the Working Mathematician. Graduate Texts in Mathematics, Vol. 5. Springer-Verlag, New York, 1971.

[12] A. Robinson, The extraordinary derived category. Math. Z. 96 (1987), no. 2, pp. 231-238.

[13] S. Schwede B. Shipley, Stable model categories are categories of modules. Topology 42 (2003), no. 1, pp. 103-153.

[14] C. A. Weibel, An Introduction to Homological Algebra. Cambridge Studies in Advanced Mathematics, vol. 38. Cambridge University Press, Cambridge, 1994.

This article may be accessed via WWW at http://www.rmi.acnet.ge/hha/ or by anonymous ftp at

ftp://ftp.rmi.acnet.ge/pub/hha/volumes/2005/n1a3/v7n1a3.(dvi,ps,pdf)

Javier J. Gutiérrez javier.gutierrez@ub.edu

Departament d'Àlgebra i Geometria

Universitat de Barcelona

Gran Via de les Corts Catalanes, 585

E-08007 Barcelona, Spain 